\title{
High Mean Arterial Pressure is Associated with Better Cognitive Function in Older Adults with Subjective Memory Complaints
}

Ada Wai Tung Fung ( $\square$ awt.fung@polyu.edu.hk)

Hong Kong Polytechnic University

Allen Ting Chun Lee

Chinese University of Hong Kong

Sukling Ma

Chinese University of Hong Kong

\section{Research Article}

Keywords: Hypertension management, Subjective memory complaints, Cognitive function, Executive Function

Posted Date: February 4th, 2021

DOl: https://doi.org/10.21203/rs.3.rs-167226/v1

License: (c) (i) This work is licensed under a Creative Commons Attribution 4.0 International License. Read Full License 


\section{Abstract}

Background: Hypertension has been shown to be a major risk factor for cognitive decline, but little is known about its association with subjective memory complaints (SMC) in late life. The study is to examine if hypertension is associated with SMC and to explore which blood pressure (BP) is more important in this group.

Methods: 497 non-demented older adults aged 60 years or older were interviewed. Hypertension is defined as a BP higher than 130/80 mmHg or on antihypertensive medication. SMC are measured by a validated 5-item Abbreviate Memory Inventory for the Chinese (AMIC) assessing subjective memory deficits in the past month. Cognitive performance was measured by a neurocognitive battery assessing global cognitive function and executive function (EF). Linear regression was performed to examine how different BP associate with AMIC and cognitive performance with adjustment for sociodemographic and physical factors.

Results: Hypertensive group had a lower AMIC than normal control $(t=-2.1, p=.033)$. Systolic blood pressure (SBP) $(B=-.008,95 \% \mathrm{Cl}-.014--.002, p=.006)$ and mean arterial pressure (MAP) $(B=-.011,95 \% \mathrm{Cl}$ $-.020--.002 p=.014)$ were associated with AMIC. SBP was only associated global cognitive function $(B=$ $.008,95 \% \mathrm{Cl} .000-.015, \mathrm{p}=.038)$, but MAP was associated with global cognitive function $(\mathrm{B}=.017,95 \% \mathrm{Cl}$ $.006-.029, \mathrm{p}=.003)$ and $\mathrm{EF}(\mathrm{B}=0.16,95 \% \mathrm{Cl} .001-.030, \mathrm{p}=.036)$.

Conclusions: High MAP was associated with better cognitive function in older adults with SMC. The findings highlight the need to be cautious when managing BP in older adults yet not to compromise their current cognitive function.

\section{Introduction}

Hypertension and cognitive decline have been extensively studied in older population, but the results are inconsistent. Traditionally, hypertension has been shown to be a major risk factor for cognitive decline in old age. High systolic blood pressure (SBP), high diastolic blood pressure (DBP), elevated SBP and DBP have all been associated with impaired cognitive performance in late life in several population based longitudinal studies (1-3). A recent population-based study in Hong Kong has also found an association between widened pulse pressure (PP) and increased risk of significant cognitive impairment in young-old adults over 6 years (4). However, accumulating epidemiological evidence has revealed that hypertension in midlife, but not late life, is a predisposing factor for cognitive decline, vascular dementia (VaD) and incident Alzheimer's disease (AD) (5). In addition, hypotension in late life have been identified as a risk factor for cognitive decline in several longitudinal studies (6-9). Therefore, evidence on treating hypertension in late life for better cognitive outcomes remains controversial.

Until recently, a randomized control trial from the Systolic Blood Pressure Intervention Trial - Memory and Cognition in Decreased Hypertension (SPRINT-MIND) provided compelling evidence on a strict blood pressure control in older adults to less than $130 / 80 \mathrm{mmHg}$ for preventing incident mild cognitive 
impairment (MCl), but not dementia, in a 4-year period (10). However, this study as well as previous studies did not completely exclude older adults with mild cognitive impairment ( $\mathrm{MCl})$ at the beginning, leading to difficulty in ruling out the influence of neurodegenerative process and reverse causality.

There is ample research evidence to suggest that brain pathology relating to hypertension may start many years before the onset of clinical impairments in dementia $(11,12)$. In order to have a clear understanding on the association between blood pressure and cognitive impairment, it would be useful to investigate hypertension in older adults with normal cognition but experiencing subjective memory complaints (SMC). This target group has relatively intact cognitive function, but not clinically demented. However, studies showed that SMC is strongly associated with substance, molecular and structural brain changes in hypertensive older adults (13-16). Therefore, hypertension might be a predisposing factor for early underlying brain changes in SMC. Currently, very little is known about how hypertension contribute to the very early subjective memory complaints (SMC) in non-demented older people who may later convert to dementia. The Hypertension in the Very Elderly Trials (HYVET) is the only study found to examine SMC in a sample of hypertensive octogenarians, but it is primarily focused on SMC conversion to dementia (17). Therefore, the aims of this study were to examine if hypertension is associated with SMC in non-demented older adults and to explore the association between different blood pressure readings and SMC. In addition, we would examine how blood pressure readings associate with cognitive function in SMC. Our result might add to the understanding on the role of hypertension in the very early stage of neurodegenerative process.

\section{Materials And Methods}

\section{Study design and participants}

A total of 497 community dwelling hypertensive older adults aged 60 or above participated in this crosssectional study from 2011 to 2013. Exclusion criteria included mild cognitive impairment (MCl), clinical dementia with a Clinical Dementia Rating (CDR) larger than or equal to 1, medical history of major depression, any conditions known to affect cognitive performance including but not limited to psychotic disorders and traumatic brain injury. $\mathrm{MCl}$ as defined by combined clinical and cognitive criterion developed in local population-based study (18). The criteria of amnesic $\mathrm{MCl}$ include: (1) presence of subjective memory complaints; (2) objective memory impairment in delayed recall score of 1.5 standard deviation (SD) below age and educational matched individuals with CDR 0; (3) relatively intact activities of daily living; and (4) absence of clinical dementia with a CDR of less than 1. Older adults possible nonamnesic $\mathrm{MCl}$ would also be recruited with the following criteria: (1) objective memory impairment in delayed recall score above - 1.5 SD cut-off; (2) impaired global cognition or other cognitive function as indicated by 1.5 SD below adjusted age and educational cut-offs; (3) 2 or more domains other than memory in CDR larger than 0 (19).

\section{Measurements}




\section{Diagnosis of hypertension}

Two blood pressure readings were taken on the right arm in a sitting position with a 15-20 minutes break in between. Hypertension is defined by two consecutive measurements with a systolic blood pressure $(\mathrm{SBP}) \geq 130 \mathrm{~mm} \mathrm{Hg}$ and/or diastolic blood pressure (DBP) $\geq 80 \mathrm{~mm} \mathrm{Hg}$, or medical history of hypertension, or on antihypertensive medication (20). Mean arterial pressure (MAP) is calculated as diastolic blood pressure plus one-third of the pulse pressure (PP), where PP refers to difference between systolic and diastolic blood pressures (21).

\section{Primary cognitive outcomes}

Subjective memory complaints (SMC) are measured by a locally validated 5-item abbreviate Memory Inventory for the Chinese (AMIC) assessing subjective cognitive deficits in everyday activities in the past month (22). The total score ranges from 0 to 5 , with higher marks indicating poor subjective cognitive functioning. A cut-off of 3 or more indicating at risk of $\mathrm{MCl}$ and early dementia.

Cognitive performance was measured by Cantonese version of the mini-mental state examination (CMMSE) (23), and a neurocognitive battery including 10-word list learning and ten-minutes delayed recall tests (24); category verbal fluency test (CVFT) (25); digit span test (26); Modified card sorting test (MCST) (27); and Chinese trail making tests ( $A$ and $B$ ) (28). For each test, the raw score was standardized into a z-score by using the age and educational adjusted mean and standard deviation (SD). Global cognitive performance was determined with a composite z-score that derived from CMMSE, CVFT, and 10-minutes delayed recall (24). Executive function included CVFT, digit span backward test, MCST and CTMT (Part B). Higher score represents better cognitive performance.

\section{Potential confounders}

Socio-demographic information include age, years of education, gender (male/ female) were obtained. Physical burdens were evaluated using the Cumulative Illness Rating Scale (CIRS) (29). Mental health status was assessed using the Revised Clinical Interview Schedule (CIS-R) that diagnosis of common mental disorders, according to the Tenth Revision of the International Classification of Diseases of the World Health Organization (ICD-10) diagnostic criteria (30). Genotyping was grouped based on the presence of Apolipoprotein e4 (ApoE4), so participants with one or more copies were categorized as e4 carrier.

\section{Statistical Analyses}

Older adults were divided into those with and without hypertension. Differences in sociodemographic and clinical profiles between the groups were compared using independent t-test for continuous score and Chi-squared test for categorical items. Linear regression was used to examine whether different blood pressure readings are associated SMC in those with hypertension and whether those blood pressure components associate with specific cognitive impairment. Four separate analyses were conducted based 
on SBP, DBP, MAP, and PP. Data analysis was performed using IBM SPSS 23.0 for Windows. Statistical significance was set at a level of 0.05 .

\section{Results}

This cross-sectional study was conducted on 497 older adults with a mean age of $68.8(S D=6.3)$ and a mean year of education of $9.8(S D=4.8)$. Slightly more than half $(54.1 \%)$ of them were females. In our sample, $69.4 \%$ had at least one SMC and $20.9 \%$ reported having three or more SMC. The sample consisted of 383 (77.1\%) individuals with hypertension. Our findings showed that, compared to those without hypertension, hypertensive group was more likely to be male $(r=12.2, p<.001)$, less educated $(t=2.0, p=.047)$, had more physical burdens $(t=-3.8, p<.001)$ and had a lower score in AMIC $(t=-2.1$, $\mathrm{p}=.033$ ). Table 1 displays the details of sociodemographic and clinical profiles between the two groups.

Linear regression was performed to examine the association between different blood pressure readings and AMIC score. After adjusting for gender, education and physical burdens, we found that both SBP $(B=-.008,95 \% \mathrm{Cl}-.014--.002, p=.006)$ and MAP $(B=-.011,95 \% \mathrm{Cl}-.020--.002 \mathrm{p}=.014)$ were significantly associated with AMIC score. We further examined the association between the above identified blood pressure readings and cognitive function. SBP was associated with global cognitive performance $(B=$ $.008,95 \% \mathrm{Cl} .000-.015, \mathrm{p}=.038)$, but not executive function $(\mathrm{B}=.003,95 \% \mathrm{Cl}-.007-.013, \mathrm{p}=.523)$. MAP was significantly associated with both global cognitive performance $(\mathrm{B}=.017,95 \% \mathrm{Cl} .006-.029, p=.003)$ and executive function $(B=0.16,95 \% \mathrm{Cl} .001-.030, p=.036)$.

\section{Discussion}

Hypertension has been shown to be a major risk factor for cognitive decline. It might also be a predisposing factor for early underlying brain changes in SMC (31). Therefore, hypertensive older adults with normal cognition but experiencing SMC is of main interests in this study, because this group concerns what constitute an effective antihypertensive treatment yet preserving their current cognitive function. In our study, we found that hypertension was associated with less SMC in a group of older adults free of $\mathrm{MCl}$ and clinical dementia. Additionally, higher MAP was significantly associated with better global cognition and executive function in hypertensive older adults. One thing to be noted is that our hypertensive group has a mean MAP value of 101.2, which is still below the range of Grade 1 hypertensive category (105.68-119 mmHg) in the MAP classification (32). This result suggest that a blood pressure control to lower than $130 / 80 \mathrm{mmHg}$ or a MAP value of $96.66 \mathrm{mmHg}$ is too tight and may have potential detrimental impact on cognitive function in older people.

Our interpretation lend supports from some longitudinal studies in oldest old (aged 85 and above) to advance the idea of a blood pressure higher than $130 / 85 \mathrm{mmHg}$ was associated with better global cognitive function $(33,34)$. Our results showed the potential role of high blood pressure, in particular MAP, on preserving cognitive function and executive function in normal older adults aged 60 and above. An explanation might be that a possible compensatory mechanism improves blood flow to the brain or 
profusion in older adults with SMC who might have long existing cerebral pathology, such as white matter lesion $(15,35)$. Previous studies have demonstrated that mean arterial pressure (MAP) is the most relevant proxy indicator that have implicated on cerebral perfusion pressure. It is also a well-established marker of cerebrovascular events, especially ischemic stroke, in both clinical and community samples $(36,37)$. However, more in-depth prospective studies with neuroimaging data must be needed to examine how high MAP may protect against early cognitive and executive function impairment relating to SMC.

Another possible explanation might be due to the use of different definitions and measurements of hypertension and executive function in previous studies. Previous studies on systemic arterial hypertension (SAH) reported poor performance in specific executive functioning tasks, such as inhibitory control and shifting (38), but some comparisons were made against normotensive younger adults. Other study found link with greater cognitive decline, but hypertension was computed as one of the aggregated vascular risks (39). HYVET is the only study found to examine SMC in a sample of hypertensive octogenarians, but it is primarily focused on SMC conversion to dementia in hypertensive older adults (17). Furthermore, SMC was measured with a single question drawn from the Geriatric Depression Scale. Their participants also had a high baseline age, in which the neurodegenerative process might already be developed over an extensive period affecting the representativeness of cognitive profiling. Furthermore, executive function was not examined in the study.

More importantly, unlike the SPRINT-MIND study, a merit in our study is the endorsement of a clear definition for SMC and $\mathrm{MCl}$ that may align future methodology for study replication, and hence result comparison. The exclusion of $\mathrm{MCl}$ and dementia in the analysis has strengthened the association identified and supported the view that a tight blood pressure control in hypertensive older adults could be associated with cognitive impairment or decline. We have also excluded those with major depression from the study and further adjusted for any mood symptoms in the analysis, so to ensure the association is independent of mood related factor. In addition, we have considered a comprehensive list of potential confounders and adjusted for factors that might affect cognitive performance.

However, a major limitation of the study is that the direction of associations regarding hypertension and SMC could not be determined. Indeed, recent neuroimaging study has drawn on the vascular hypothesis of cognitive decline, by which suggest that long-term exposure to hypertension may lead to hypoperfusion of the white matter predisposing early manifestation of subclinical AD (15). However, we lack direct neuroimaging data to support hypertension as a contributing factor to the association between cerebral pathology and SMC. We also lack data on the duration of hypertension, so effect of midlife hypertension on current cognitive performance was not known.

\section{Conclusions}

Our results showed that hypertension is associated with SMC in older adults, but screening for cerebral pathology in older adults with SMC is still debatable not only for its uncertainty as a diagnostic tool, but also for being an invasive and expensive means. Our study might provide useful prognostic information 
in hypertensive older adults experiencing SMC, and perhaps a close MAP monitoring is more relevant and practical for preventing cognitive decline in this high-risk group. Prospective studies in the future is needed to get more inference on causation, especially on the relationship between MAP and development of executive dysfunction older adults with SMC. This will also help to support if changes in MAP might reflect a very early stage of neurodegeneration.

\section{Declarations}

\section{Ethical approval and consent to participate}

The authors assert that all procedures contributing to this work comply with the ethical standards of the relevant national and institutional committees on human experimentation and with the Helsinki

Declaration of 1975, as revised in 2008. All procedures involving human subjects/patients were approved by the Clinical Research Ethics Committee of the Chinese University of Hong Kong (Project Code: 8303102). Written informed consent was obtained from all subjects before testing.

\section{Consent for publication}

Not applicable.

\section{Availability of data and materials}

The dataset generated and/or analyzed during the current study are not publicly available due privacy but are available from the corresponding author on reasonable request.

\section{Competing interests}

The authors declare that they have no competing interests.

\section{Funding}

The study was supported by the Lui Che Woo Institute of Innovative Medicine (Project Code: 8303102). The funding body has no role in design of the study and collection, analysis, and interpretation of data and in writing the manuscript.

\section{Author contributions}

AWTF formulated the research question, involved in the study design, data collection, data analysis and interpretation, writing and editing of manuscript. SLM involved in genetic data analysis and commenting 
on manuscript. ATCL involved in critical commenting on study design, statistical analysis and manuscript writing.

\section{Acknowledgements}

Not applicable.

\section{References}

1. Elias MF, Wolf PA, D'Agostino RB, Cobb J, White LR. Untreated blood pressure level is inversely related to cognitive functioning: the Framingham Study. Am J Epidemiol. 1993;138(6):353-64.

2. Launer LJ, Masaki K, Petrovitch H, Foley D, Havlik RJ. The Association Between Midlife Blood Pressure Levels and Late-Life Cognitive Function: The Honolulu-Asia Aging Study. JAMA. 1995;274(23):1846-51.

3. Kilander L, Nyman $H$, Boberg M, Hansson L, Lithell $H$. Hypertension is related to cognitive impairment: a 20-year follow-up of 999 men. Hypertension. 1998;31(3):780-6.

4. Lee AT, Chan WC, Chiu HF, Richards M, Ng SP, Hui LY, et al. Widened pulse pressure is a potential risk factor for significant cognitive impairment among community-dwelling Chinese younger old people. J Alzheimers Dis. 2013;35(4):687-96.

5. Iadecola C, Gottesman RF. Neurovascular and Cognitive Dysfunction in Hypertension. Circ Res. 2019;124(7):1025-44.

6. Borenstein AR, Wu Y, Mortimer JA, Schellenberg GD, McCormick WC, Bowen JD, et al. Developmental and vascular risk factors for Alzheimer's disease. Neurobiol Aging. 2005;26(3):325-34.

7. Petitti DB, Crooks VC, Buckwalter JG, Chiu V. Blood pressure levels before dementia. Arch Neurol. 2005;62(1):112-6.

8. Kuller LH, Lopez OL, Newman A, Beauchamp NJ, Burke G, Dulberg C, et al. Risk factors for dementia in the cardiovascular health cognition study. Neuroepidemiology. 2003;22(1):13-22.

9. Lindsay J, Laurin D, Verreault R, Hébert R, Helliwell B, Hill GB, et al. Risk factors for Alzheimer's disease: a prospective analysis from the Canadian Study of Health and Aging. Am J Epidemiol. 2002;156(5):445-53.

10. Williamson JD, Pajewski NM, Auchus AP, Bryan RN, Chelune G, Cheung AK, et al. Effect of Intensive vs Standard Blood Pressure Control on Probable Dementia: A Randomized Clinical Trial. Jama. 2019;321(6):553-61.

11. Qiu C, Winblad B, Fratiglioni L. The age-dependent relation of blood pressure to cognitive function and dementia. Lancet Neurol. 2005;4(8):487-99.

12. Elias PK, Elias MF, Robbins MA, Budge MM. Blood pressure-related cognitive decline: does age make a difference? Hypertension. 2004;44(5):631-6. 
13. Schultz SA, Oh JM, Koscik RL, Dowling NM, Gallagher CL, Carlsson CM, et al. Subjective memory complaints, cortical thinning, and cognitive dysfunction in middle-age adults at risk of $A D$.

Alzheimer's \& Dementia : Diagnosis, Assessment \& Disease Monitoring. 2015;1(1):33-40.

14. Buckley RF, Hanseeuw B, Schultz AP, Vannini P, Aghjayan SL, Properzi MJ, et al. Region-Specific Association of Subjective Cognitive Decline With Tauopathy Independent of Global beta-Amyloid Burden. JAMA Neurol. 2017;74(12):1455-63.

15. Kearney-Schwartz A, Rossignol P, Bracard S, Felblinger J, Fay R, Boivin JM, et al. Vascular structure and function is correlated to cognitive performance and white matter hyperintensities in older hypertensive patients with subjective memory complaints. Stroke. 2009;40(4):1229-36.

16. Minett TSC, Dean JL, Firbank M, English P, O'Brien JT. Subjective Memory Complaints, White-Matter Lesions, Depressive Symptoms, and Cognition in Elderly Patients. The American Journal of Geriatric Psychiatry. 2005;13(8):665-71.

17. Peters R, Beckett N, Antikainen R, Rockwood K, Bulpitt CJ, Anstey KJ. Subjective memory complaints and incident dementia in a high risk older adult hypertensive population. Age Ageing. 2019;48(2):253-9.

18. Lam LC, Tam CW, Leung GT, Lui VW, Fung AW, Chiu HF, et al. Combined clinical and cognitive criteria to identify mild cognitive impairment in a southern Chinese community. Alzheimer Dis Assoc Disord. 2010;24(4):343-7.

19. Morris JC. Clinical dementia rating: a reliable and valid diagnostic and staging measure for dementia of the Alzheimer type. Int Psychogeriatr. 1997;9 Suppl 1:173-6; discussion 7-8.

20. Kario K, Shin J, Chen CH, Buranakitjaroen P, Chia YC, Divinagracia R, et al. Expert panel consensus recommendations for ambulatory blood pressure monitoring in Asia: The HOPE Asia Network. J Clin Hypertens (Greenwich). 2019;21(9):1250-83.

21. WA. B. Chapter 16. Blood Pressure. . In: Walker HK HW, Hurst JW, editor. Clinical Methods: The History, Physical, and Laboratory Examinations. 3rd edition ed. Boston: Butterworths; 1990.

22. Lam LC, Lui VW, Tam CW, Chiu HF. Subjective memory complaints in Chinese subjects with mild cognitive impairment and early Alzheimer's disease. Int J Geriatr Psychiatry. 2005;20(9):876-82.

23. Chiu H, Lee H, Chung W, Kwong P. Reliability and validity of the Cantonese version of the Mini-Mental State Examination - a preliminary study. Journal of Hong Kong College of Psychiatry. 1994;4(SP2):25-8.

24. Chu LW, Chiu KC, Hui SL, Yu GK, Tsui WJ, Lee PW. The reliability and validity of the Alzheimer's Disease Assessment Scale Cognitive Subscale (ADAS-Cog) among the elderly Chinese in Hong Kong. Ann Acad Med Singapore. 2000;29(4):474-85.

25. Chiu HF, Chan CK, Lam LC, Ng KO, Li SW, Wong M, et al. The modified Fuld Verbal Fluency Test: a validation study in Hong Kong. J Gerontol B Psychol Sci Soc Sci. 1997;52(5):P247-50.

26. Elwood RW. The Wechsler Memory Scale-Revised: Psychometric characteristics and clinical application. Neuropsychology Review. 1991;2(2):179-201. 
27. Chan CWY, Lam LCW, Wong TCM, Chiu HFK. Modified Card Sorting Test Performance among Community Dwelling Elderly Chinese People. Hong Kong Journal of Psychiatry. 2003;13:2-7+29.

28. Bowie CR, Harvey PD. Administration and interpretation of the Trail Making Test. Nat Protoc. 2006;1(5):2277-81.

29. Parmelee PA, Thuras PD, Katz IR, Lawton MP. Validation of the Cumulative Illness Rating Scale in a geriatric residential population. J Am Geriatr Soc. 1995;43(2):130-7.

30. Lewis G, Pelosi AJ, Araya R, Dunn G. Measuring psychiatric disorder in the community: a standardized assessment for use by lay interviewers. Psychol Med. 1992;22(2):465-86.

31. Faraco G, ladecola C. Hypertension: a harbinger of stroke and dementia. Hypertension. 2013;62(5):810-7.

32. Nath Kundu R, Biswas, S., \& Das, M. . Mean Arterial Pressure Classification: A Better Tool for Statistical Interpretation of Blood Pressure Related Risk Covariates. . Cardiology and Angiology: An International Journal. 2017;6(1): 1-7.

33. Harrison SL, Stephan BC, Siervo M, Granic A, Davies K, Wesnes KA, et al. Is there an association between metabolic syndrome and cognitive function in very old adults? The Newcastle 85+ Study. J Am Geriatr Soc. 2015;63(4):667-75.

34. Euser SM, van Bemmel T, Schram MT, Gussekloo J, Hofman A, Westendorp RG, et al. The effect of age on the association between blood pressure and cognitive function later in life. J Am Geriatr Soc. 2009;57(7):1232-7.

35. Yaffe K. Prevention of Cognitive Impairment With Intensive Systolic Blood Pressure Control. JAMA. 2019;321(6):548-9.

36. Verdecchia P, Schillaci G, Reboldi G, Franklin Stanley S, Porcellati C. Different Prognostic Impact of 24-Hour Mean Blood Pressure and Pulse Pressure on Stroke and Coronary Artery Disease in Essential Hypertension. Circulation. 2001;103(21):2579-84.

37. Zheng L, Sun Z, Li J, Zhang R, Zhang X, Liu S, et al. Pulse pressure and mean arterial pressure in relation to ischemic stroke among patients with uncontrolled hypertension in rural areas of China. Stroke. 2008;39(7):1932-7.

38. Moraes NC, Aprahamian I, Yassuda MS. Executive function in systemic arterial hypertension: A systematic review. Dementia \& neuropsychologia. 2019;13(3):284-92.

39. Kaffashian S, Dugravot A, Elbaz A, Shipley MJ, Sabia S, Kivimaki M, et al. Predicting cognitive decline: a dementia risk score vs. the Framingham vascular risk scores. Neurology. 2013;80(14):1300-6.

\section{Table}


Table 1

Sociodemographic and clinical profile of those with and without hypertension.

\begin{tabular}{|c|c|c|c|c|c|}
\hline & \multicolumn{2}{|c|}{$\begin{array}{l}\text { Hypertension } \\
(\geq 130 / 80 \mathrm{mmHg}) \\
(\mathrm{N}=388)\end{array}$} & \multicolumn{2}{|c|}{$\begin{array}{l}\text { No hypertension }(<130 / 80 \mathrm{mmHg}) \\
(\mathrm{N}=115)\end{array}$} & $\begin{array}{l}\text { T-test / } \\
\text { Cross Tab }\end{array}$ \\
\hline & $M$ & SD & M & SD & $p$-value \\
\hline Age & 69.1 & 6.4 & 68.0 & 6.0 & .096 \\
\hline Male (\%) & 50.1 & - & 31.6 & - & $<.001$ \\
\hline Years of Education & 9.6 & 4.8 & 10.6 & 4.5 & .047 \\
\hline CIRS & 4.3 & 2.4 & 3.5 & 1.9 & $<.001$ \\
\hline CISR & 2.7 & 4.3 & 3.7 & 5.5 & .068 \\
\hline SBP & 139.2 & 19.2 & 116.4 & 8.5 & $<.001$ \\
\hline DBP & 82.2 & 11.8 & 70.8 & 6.9 & $<.001$ \\
\hline MAP & 101.2 & 12.5 & 86.0 & 6.5 & $<.001$ \\
\hline PP & 57.0 & 16.2 & 45.6 & 8.0 & $<.001$ \\
\hline CMMSE & 28.6 & 1.4 & 28.9 & 1.4 & .121 \\
\hline AMIC & 1.4 & 1.3 & 1.7 & 1.4 & .033 \\
\hline ApoE 4 carriers $(\%)$ & 16.5 & - & 18.5 & - & .658 \\
\hline \multicolumn{6}{|c|}{$\begin{array}{l}\text { Note: } \mathrm{NC}=\text { Normal cognition; } \mathrm{MCl}=\text { Mild cognitive impairment; } \mathrm{M}=\text { Mean; } \mathrm{SD}=\text { Standard deviation; } \mathrm{CIRS=} \\
\text { Chronic Illness Rating Scale; } \mathrm{CISR}=\mathrm{Revised} \text { Clinical Interview Schedule; SBP= Systolic Blood Pressure, } \\
\text { DBP= Diastolic Blood Pressure; MAP = Mean Arterial Pressure; } \mathrm{PP}=\mathrm{Pul} \text { = Pressure; } \\
\text { CMMSE=Cantonese version of the mini-mental state examination; AMIC= Abbreviated Memory } \\
\text { Inventory for the Chinese. }\end{array}$} \\
\hline
\end{tabular}

